Enferm Bras 2019;18(1);65-74

http://dx.doi.org/10.33233/eb.v18i1.2457

\title{
ARTIGO ORIGINAL \\ Conhecimento de enfermeiros sobre os diferentes estágios clínicos da sepse: estudo descritivo
}

Yara Gouvea Areal*, Luana Vieira Toledo, M.Sc. ${ }^{* \star}$, Cristiane Chaves de Souza, D.Sc. ${ }^{* \star}$, Tiago Ricardo Moreira, D.Sc. ${ }^{* \star *}$, Camila Santana Domingos, M.Sc. ${ }^{* \star *}$, Patrícia de Oliveira Salgado, D.Sc. ${ }^{* * * * *}$

*Acadêmica do curso de graduação em Enfermagem, Departamento de Medicina e Enfermagem da Universidade Federal de Viçosa (UFV), Viçosa/MG, ${ }^{* * P}$ rofessora Assistente do Departamento de Medicina e Enfermagem da Universidade Federal de Viçosa (UFV), Viçosa/MG, ${ }^{* * *}$ Professora Adjunto do Departamento de Medicina e Enfermagem da Universidade Federal de Viçosa (UFV), Viçosa/MG, ${ }^{* * * *}$ Professor Adjunto do Departamento de Medicina e Enfermagem da Universidade Federal de Viçosa (UFV), Viçosa/MG, ${ }^{\star * * \star \star E n f e r m e i r a ~}$ do Departamento de Medicina e Enfermagem da Universidade Federal de Viçosa (UFV), Viçosa/MG, ${ }^{* * * * * *}$ Professora Adjunto do Departamento de Medicina e Enfermagem da Universidade Federal de Viçosa (UFV), Viçosa/MG

Recebido em 17 de julho de 2018; aceito em 1 de outubro de 2018.

Endereço de correspondência: Yara Gouvea Areal, Travessa César Santana, 09/103, Centro, 36570-000 Viçosa/MG, E-mail: yara.areal@hotmail.com; Luana Vieira Toledo: luanatoledoufv@gmail.com; Cristiane Chaves de Souza: cristiane.chaves@ufv.br; Tiago Ricardo Moreira: tiagoricardomoreira@gmail.com; Camila Santana Domingos: camilasantanadomingos@gmail.com; Patrícia de Oliveira Salgado: patriciaoliveirasalgado@gmail.com

\section{Resumo}

Introdução: A Sepse é definida como uma resposta sistêmica a uma doença infecciosa, cuja incidência vem aumentando entre os indivíduos. Para os pacientes sépticos uma adequada assistência de enfermagem, baseada em conhecimento e habilidades técnico-científicas, possui um papel fundamental na evolução positiva do quadro clínico. Objetivo: Identificar o conhecimento de enfermeiros sobre os diferentes estágios clínicos da sepse. Métodos: Estudo descritivo, realizado entre março e maio de 2017 com enfermeiros de uma unidade hospitalar da Zona da Mata Mineira. Os dados foram coletados utilizando questionário semiestruturado com questões no formato de caso clínico abordando o conhecimento dos diferentes estágios clínicos da sepse e a identificação dos seus sinais e sintomas. Utilizou-se estatística descritiva para análise dos dados. Resultados: Dos 23 enfermeiros entrevistados, 19 (82,6\%) afirmaram ter conhecimento moderado sobre a temática. Em relação ao papel da faculdade na troca de conhecimentos, $15(65,2 \%)$ enfermeiros alegaram que pouco conhecimento foi adquirido enquanto graduandos. Apenas $10(43,5 \%)$ enfermeiros identificaram corretamente algum dos casos clínicos relacionado à sepse. Conclusão: Há dificuldade na aplicabilidade clínica dos conceitos da sepse e seus estágios clínicos pelos enfermeiros, o que contribui para um atraso na identificação precoce dos casos e piora do prognóstico.

Palavras-chave: sepse, enfermagem, conhecimento, saúde pública.

\footnotetext{
Abstract

Knowledge of nurses about different clinical stages of sepsis: descriptive study

Introduction: Sepsis is defined as a systemic response to an infectious disease, the incidence of which is increasing among individuals. For septic patients an adequate nursing care, based on knowledge and technical-scientific skills, plays a fundamental role in the positive evolution of the clinical picture. Objective: To identify the knowledge of nurses about the different clinical stages of sepsis. Methods: Descriptive study, conducted between March and May 2017 with nurses from a hospital unit in the Zona da Mata Mineira. The data were collected using a semistructured questionnaire with questions in the clinical case format about the knowledge of the different clinical stages of sepsis and the identification of its signs and symptoms.
} 
Descriptive statistics were used to analyze the data. Results: Of the 23 nurses interviewed, 19 $(82.6 \%)$ stated that they had moderate knowledge on the subject. Regarding the role of faculty in the exchange of knowledge, $15(65.2 \%)$ nurses claimed that little knowledge was acquired while undergraduates. Only $10(43.5 \%)$ nurses correctly identified any of the clinical cases related to sepsis. Conclusion: There is difficulty in the clinical applicability of the concepts of sepsis and its clinical stages by nurses, which contributes to a delay in the early identification of the cases and worsening of the prognosis.

Key-words: sepsis, nursing, knowledge, public health.

\section{Resumen \\ Conocimiento de enfermeros sobre los diferentes estadios clínicos de sepsis: estudio descriptivo}

Introducción: La sepsis se define como una respuesta sistémica a una enfermedad infecciosa, cuya incidencia viene aumentando entre los individuos. Para los pacientes sépticos una adecuada asistencia de enfermería, basada en conocimiento y habilidades técnico-científicas, tiene un papel fundamental en la evolución positiva del cuadro clínico. Objetivo: Identificar el conocimiento de enfermeros sobre las diferentes etapas clínicas de la sepsis. Métodos: Estudio descriptivo, realizado entre marzo y mayo de 2017 con enfermeros de una unidad hospitalaria de la Zona de la Mata Minera. Los datos fueron recolectados utilizando cuestionario semiestructurado con cuestiones en el formato de caso clínico abordando el conocimiento de los diferentes estadios clínicos de la sepsis y la identificación de sus signos y síntomas. Se utilizó estadística descriptiva para el análisis de los datos. Resultados: De los 23 enfermeros entrevistados, $19(82,6 \%)$ afirmaron tener conocimiento moderado sobre la temática. En cuanto al papel de la facultad en el intercambio de conocimientos, $15(65,2 \%)$ enfermeros alegaron que poco conocimiento fue adquirido como graduandos. Sólo $10(43,5 \%)$ enfermeros identificaron correctamente alguno de los casos clínicos relacionados con la sepsis. Conclusión: Hay dificultad en la aplicabilidad clínica de los conceptos de la sepsis y sus etapas clínicas por los enfermeros, lo que contribuye a un retraso en la identificación precoz de los casos y empeoramiento del pronóstico.

Palabras-clave: sepsis, enfermería, conocimiento, salud pública.

Introdução

A Sepse é definida como uma resposta sistêmica a uma doença infecciosa, cuja incidência vem aumentando entre os indivíduos. Este aumento pode estar relacionado ao envelhecimento da população, ao crescimento da resistência bacteriana, ou à melhoria no atendimento dos serviços de emergência, fazendo com que mais pacientes graves sobrevivam ao insulto inicial [1]. O aumento dos casos de sepse vem gerando impacto no sistema de saúde, marcado pelos elevados custos decorrentes das internações hospitalares, especialmente nas Unidades de Terapia Intensiva (UTI), como também pela grande mortalidade [2].

Embora os dados epidemiológicos relacionados à sepse sejam escassos ou inexistentes para os países em desenvolvimento, estudos estimam que esta seja uma das principais causas de morte do mundo [3-4]. A sepse apresenta-se como uma das grandes preocupações da saúde pública, representando mais de US\$20 bilhões do total de custos dos hospitais nos EUA, sendo a principal anormalidade fisiológica, patológica e bioquímica induzida por infecção [2]. O estudo Sepsis Prevalence Assessment Database (SPREAD), conduzido pelo Instituto Latino Americano da Sespe (ILAS) em 229 UTI's brasileiras selecionadas aleatoriamente para representarem, de maneira adequada, o cenário nacional, apontou que $30 \%$ dos leitos de UTI do país estão ocupados por pacientes com sepse ou choque séptico. A letalidade nesses pacientes foi de 55\% [4].

No Brasil, um estudo realizado em duas UTI de um Hospital Universitário de São Paulo no ano de 2013 identificou a sepse como sendo a principal causa de mortalidade dos pacientes internados. Dentre as causas do óbito, o choque séptico foi prevalente em mais da metade dos indivíduos, o que reforça a magnitude da doença [5].

Por ser fundamentalmente clínico, o diagnóstico de sepse apresenta-se como um desafio para os profissionais de saúde, devido à necessidade de reconhecimento imediato e tratamento precoce, aliado à dificuldade de identificação dos sintomas, sobretudo por parte dos enfermeiros. Comumente, o diagnóstico de sepse é feito tardiamente, pois os sinais e sintomas como alteração na contagem de leucócitos, febre, taquicardia e taquipneia não são específicos 
e podem subestimar um quadro de sepse, propiciando a evolução para estágios mais graves da doença $[3,6,7]$.

A sepse pode se manifestar em diferentes estágios clínicos, sendo a sua gravidade diretamente proporcional à evolução temporal da doença. Inicialmente apresenta-se como um quadro de Síndrome da Resposta Inflamatória Sistêmica (SIRS) associado a uma infecção. Progressivamente pode evoluir para casos de sepse grave, quando há algum quadro de disfunção orgânica e, quando não tratado precocemente, pode culminar em um estado de choque séptico, caracterizado por hipotensão persistente, mesmo após infusão de grande quantidade de volume [8].

Nesse sentido, para que haja a identificação precoce dos sinais e sintomas de sepse e seja delineada uma assistência efetiva em conformidade com as necessidades dos pacientes, os profissionais de enfermagem precisam aliar os conhecimentos científicos à sua prática clínica [9-10]. Entretanto, observa-se que há uma fragilidade dos enfermeiros na aplicabilidade do conhecimento científico nos contextos assistenciais. Um estudo realizado em Rondônia identificou que os enfermeiros entrevistados conheciam o conceito de sepse, no entanto, quando questionados sobre as intervenções necessárias para a melhoria no quadro de sepse baseada em evidências clínicas, observou-se um déficit de conhecimento por parte dos enfermeiros [11]. Essa deficiência de conhecimento pode estar relacionada a um comprometimento da assistência ao paciente com sepse, que pode resultar em uma evolução clínica negativa e consequente aumento da mortalidade por esta condição.

Tendo em vista que a sepse é uma síndrome com impacto mundial e entendendo que uma adequada assistência de enfermagem, baseada em conhecimento e habilidades técnicocientíficas, possui um papel fundamental na evolução positiva do paciente séptico, justifica-se a realização desta pesquisa. A identificação do nível de conhecimento dos profissionais de enfermagem poderá contribuir para o planejamento de ações visando à melhoria dos cuidados oferecidos aos pacientes com sepse, com vistas à identificação precoce dos casos.

Com base no exposto, este estudo teve como objetivo identificar o conhecimento de enfermeiros sobre os diferentes estágios clínicos da sepse.

Material e métodos

Trata-se de estudo descritivo, realizado no período de março a maio de $2017 \mathrm{com}$ enfermeiros vinculados a uma unidade hospitalar localizada em um município de médio porte da Zona da Mata Mineira. O referido Hospital é uma instituição de caráter assistencial, sem fins lucrativos e que tem por finalidade a prestação de assistência médico-hospitalar em situações clínicas e cirúrgicas.

A população do estudo foi composta por todos os enfermeiros com vínculo empregatício no período de coleta de dados $(\mathrm{N}=30)$. Incluíram-se todos os enfermeiros portadores de diploma de graduação em instituição reconhecida pelo Ministério da Educação e Cultura. Foram excluídos aqueles enfermeiros que estavam afastados do serviço por qualquer motivo durante o período de realização das entrevistas. A amostra final, definida por conveniência, foi composta por 23 enfermeiros.

Os dados foram coletados a partir da utilização de um questionário semiestruturado construído e validado por outras pesquisadoras, mediante o consentimento e a autorização das mesmas [12]. O referido questionário, utilizado em uma pesquisa com discentes do curso de graduação em enfermagem, possuía duas partes, sendo a primeira referente à caracterização da população estudada, para obtenção de dados relativos à idade, título de especialização, tempo de formação e tempo de atuação no atual setor do hospital. A segunda parte continha questões objetivas e de múltiplas que permitiu aos participantes informarem sobre a presença ou não de conhecimento teórico sobre a sepse. A primeira questão teve por objetivo identificar, com base em uma autoavaliação, o nível de conhecimento que os enfermeiros possuíam sobre a sepse. A segunda questão destinou-se a avaliar, na visão dos participantes, se houve uma troca de conhecimentos sobre a temática durante a graduação. Em ambas as questões as alternativas de resposta variavam entre nenhum, pouco, moderado e muito. Sequencialmente apresentaram-se quatro questões no formato de casos clínicos em que o participante deveria identificar se a situação clínica descrita correspondia a um quadro de SIRS, sepse, sepse grave ou choque séptico. Por fim, ao final do questionário foram incluídas duas tabelas contendo os sinais/sintomas do paciente com sepse e sepse grave, em que, para cada sinal/sintoma, o enfermeiro deveria indicar com as respostas "sim", "não" ou "não sei" se o mesmo era pertinente aos quadros de sepse e sepse grave [12]. 
Destaca-se que as pesquisadoras responsáveis pela construção do questionário utilizaram como referência os dados de identificação dos sinais e sintomas da sepse contido nas diretrizes internacionais de 2008. Para a obtenção dos dados neste estudo, as tabelas de sinais e sintomas da sepse e sepse grave foram atualizadas conforme as Diretrizes internacionais para tratamento de sepse grave e choque séptico do ano de 2012.

Para a coleta de dados agendou-se, previamente, um encontro, no próprio hospital, com os participantes do estudo para que respondessem ao questionário. Para a preservação do anonimato dos enfermeiros, os questionários foram identificados pela letra $E$, acompanhados pelo número correspondente à ordem de realização da entrevista, a saber: E1, E2, E3.

Os dados foram analisados utilizando estatística descritiva no programa Excel 2010. As frequências absoluta e relativa de cada variável de interesse do estudo foram expressas em tabelas.

O estudo foi aprovado pelo Comitê de Ética em Pesquisa com Seres Humanos da Universidade Federal de Viçosa (Parecer: 1.668.556). A pesquisa foi desenvolvida respeitandose os aspectos éticos da pesquisa com seres humanos conforme a Resolução 466/2012 do Conselho Nacional de Saúde. Os enfermeiros que atenderam aos critérios de inclusão foram esclarecidos quanto aos objetivos da pesquisa, convidados a participar do estudo e, aos que aceitaram, foi solicitada a assinatura do Termo de Consentimento Livre e Esclarecido (TCLE).

\section{Resultados}

Dos 23 enfermeiros entrevistados, $16(69,6 \%)$ eram do sexo feminino. A média de idade foi de 34,7 anos (26-60 anos). Em relação ao tempo de formação, a maioria (14 - 60,9\%) havia completado o curso de graduação entre cinco e 10 anos, e $17(73,9 \%)$ já tinham concluído no mínimo um curso de especialização. Quanto ao setor de atuação, a maior parte dos enfermeiros (9-43,5\%) atuava em enfermarias e possuía um vínculo com o setor há menos de 12 meses (13-56,5\%) conforme exposto na Tabela I.

Tabela I - Caracterização da população estudada, segundo sexo, idade, tempo de formação, curso de especialização e tempo de atuação no setor. Viçosa/MG, Brasil, 2017. ( $n=23)$

\begin{tabular}{lll}
\hline Variáveis & Frequências de distribuição \\
\hline & $\mathbf{n}$ & $\%$ \\
\hline Sexo & 16 & 69,6 \\
Meminino & 7 & 30,4 \\
Masculino & 6 & 26,1 \\
$<30$ anos & 12 & 52,2 \\
30 a 40 anos & 4 & 17,4 \\
40 a 50 anos & 1 & 4,3 \\
50 a 60 anos & 1 & 4,3 \\
Tempo de formação & 6 & 26,1 \\
$<1$ ano & 14 & 60,9 \\
1 a 5 anos & 2 & 8,7 \\
5 a 10 anos & & \\
$>10$ anos & 17 & 73,9 \\
Curso de especialização & 6 & 26,1 \\
Sim & & \\
Não & 10 & 43,5 \\
Setor de atuação & 6 & 26,1 \\
Enfermarias & 4 & 17,4 \\
Unidades de Terapia Intensiva & 3 & 13,0 \\
Urgência e Emergência & & \\
Centro Cirúrgico/Controle de Infecção & 13 & 56,5 \\
Tempo de atuação no setor & 5 & 21,8 \\
$<12$ meses & 4 & 17,4 \\
12 a 24 meses & 1 & 4,3 \\
25 a 36 meses & &
\end{tabular}

Fonte: Dados da pesquisa. 
Em relação ao conhecimento que os enfermeiros afirmam possuir em relação à sepse, seus sinais e sintomas e complicações, percebeu-se que apenas quatro $(17,4 \%)$ referiram pouco conhecimento, e $19(82,6 \%)$ afirmaram ter conhecimento moderado. Não foram identificados enfermeiros que desconheciam ou que possuíam muito conhecimento sobre a sepse.

Quanto ao papel da graduação enquanto instituição formadora e promotora de conhecimentos relacionados à sepse, apenas um enfermeiro $(4,3 \%)$ relatou que não obteve qualquer conhecimento sobre sepse enquanto graduando, $15(65,2 \%)$ afirmaram que receberam pouco conhecimento, cinco $(21,7 \%)$ julgaram o conhecimento obtido na graduação como moderado, e dois $(8,7 \%)$ alegaram ter recebido muito conhecimento sobre a sepse enquanto frequentaram a academia.

No que tange à identificação de SIRS, Sepse, Sepse Grave e Choque Séptico a partir da utilização de casos clínicos e classificação adequada dos pacientes, percebeu-se que houve um número expressivo de erros, sobretudo na identificação da sepse grave (19-82,6\%) e sepse $(69,6 \%)$. A SIRS foi a síndrome identificada corretamente pelo maior número de enfermeiros $(10-43,5 \%)$, seguida do choque séptico $(8-34,8 \%)$. Vale destacar que uma parcela dos enfermeiros não soube identificar os casos de SIRS (3-13,0\%), sepse (3-13,0\%) e choque séptico (1-4,3\%) (tabela II).

Tabela II - Conhecimento dos enfermeiros sobre a definição de Síndrome da Resposta Inflamatória Sistêmica, sepse, sepse grave e choque séptico. Viçosa, MG, Brasil, 2017. ( $n=23)$

\begin{tabular}{|c|c|c|c|c|c|c|c|}
\hline \multirow{2}{*}{$\begin{array}{l}\text { Conhecimento das } \\
\text { definições }\end{array}$} & & \multicolumn{2}{|c|}{ Acertos } & \multicolumn{2}{|c|}{ Erros } & \multicolumn{2}{|c|}{ Não Sei } \\
\hline & & $n$ & $\%$ & $n$ & $\%$ & $\mathbf{n}$ & $\%$ \\
\hline $\begin{array}{lll}\text { Síndrome } & \text { da } & \text { Resposta } \\
\text { Sistêmica } & & \\
\end{array}$ & Inflamatória & 10 & 43,5 & 10 & 43,5 & 3 & 13,0 \\
\hline Sepse & & 4 & 17,4 & 16 & 69,6 & 3 & 13,0 \\
\hline Sepse grave & & 4 & 17,4 & 19 & 82,6 & 0 & 0,0 \\
\hline Choque séptico & & 8 & 34,8 & 14 & 60,9 & 1 & 4,3 \\
\hline
\end{tabular}

Fonte: Dados da pesquisa.

Quanto ao reconhecimento dos sintomas da sepse e sepse grave, observou-se que os enfermeiros relacionaram tais quadros clínicos com a confirmação de infecção, sendo este o sintoma mais relatado tanto na sepse $(21-91,3 \%)$ quanto na sepse grave $(23-100,0 \%)$. Destaca-se também a questão da temperatura corporal, grande parte dos enfermeiros (20$86,9 \%)$ reconheceu a elevação da temperatura $\left(>38^{\circ} \mathrm{C}\right)$ como um sinal sugestivo de sepse e sepse grave. No entanto, desconsideraram a redução da temperatura $\left(<36^{\circ} \mathrm{C}\right)$ como uma variável importante a ser analisada. Outros sinais e sintomas que mereceram atenção devido à baixa correlação por parte dos enfermeiros ao quadro de sepse referem-se à alteração da Frequência Cardíaca (>90bpm) e a suspeita de infecção, as quais foram identificadas respectivamente por apenas $11(47,8 \%)$ e $10(43,5 \%)$ dos enfermeiros. Nos casos de sepse grave, observou-se que apenas $11(47,8 \%)$ enfermeiros apontaram nível glicêmico $>110 \mathrm{mg} / \mathrm{dl}$ como um fator de risco. As tabelas III e IV apresentam o total de respostas dos enfermeiros para a identificação dos sinais e sintomas relativos ao quadro de sepse e sepse grave.

Tabela III - Conhecimento dos enfermeiros sobre os sinais e sintomas sugestivos de sepse. Viçosa, MG, Brasil, 2017. ( $n=23)$

\begin{tabular}{|c|c|c|c|c|c|c|}
\hline \multirow[b]{2}{*}{ Sinais e Sintomas } & \multicolumn{2}{|c|}{ Sim } & \multicolumn{2}{|c|}{ Não } & \multicolumn{2}{|c|}{ Não sei } \\
\hline & $\mathbf{n}$ & $\%$ & $\mathbf{n}$ & $\%$ & $\mathbf{n}$ & $\%$ \\
\hline Temperatura corporal $>38^{\circ} \mathrm{C}$ & 20 & 86,9 & 3 & 13,1 & 0 & 0,0 \\
\hline Temperatura corporal $<36^{\circ} \mathrm{C}$ & 3 & 13,0 & 19 & 82,6 & 1 & 4,4 \\
\hline Frequência cardíaca > 90 bpm & 11 & 47,8 & 12 & 52,2 & 0 & 0,0 \\
\hline Frequência respiratória>20lrpm* & 16 & 69,6 & 4 & 17,3 & 2 & 8,7 \\
\hline Infecção confirmada & 21 & 91,3 & 2 & 8,7 & 0 & 0,0 \\
\hline Suspeita de infecção & 10 & 43,5 & 10 & 43,5 & 3 & 13 \\
\hline
\end{tabular}

*Um dos participantes não respondeu a essa questão. Fonte: Dados da pesquisa. 
Tabela IV - Conhecimento dos enfermeiros sobre os sinais e sintomas sugestivos de sepse grave. Viçosa, MG, Brasil, 2017. ( $n=23)$

\begin{tabular}{|c|c|c|c|c|c|c|}
\hline \multirow[b]{2}{*}{ Sinais e Sintomas } & \multicolumn{2}{|c|}{ Sim } & \multicolumn{2}{|c|}{ Não } & \multicolumn{2}{|c|}{ Não sei } \\
\hline & $\mathbf{n}$ & $\%$ & $\mathbf{n}$ & $\%$ & $\mathbf{n}$ & $\%$ \\
\hline Temperatura corporal $>38^{\circ} \mathrm{C}^{*}$ & 20 & 86,9 & 2 & 8,7 & 0 & 0,0 \\
\hline Temperatura corporal $<36^{\circ} \mathrm{C}^{*}$ & 4 & 17,3 & 17 & 73,9 & 1 & 4,4 \\
\hline Frequência cardíaca >90bpm & 18 & 78,3 & 4 & 17,3 & 1 & 4,4 \\
\hline Frequência respiratória >20 Irpm** & 19 & 82,6 & 2 & 8,7 & 0 & 0,0 \\
\hline Infecção confirmada & 23 & 100,0 & 0 & 0,0 & 0 & 0,0 \\
\hline $\begin{array}{l}\text { Glicemia capilar }>110 \mathrm{mg} / \mathrm{dl} \text { em } \\
\text { pacientes sem diabetes mellitus }\end{array}$ & 11 & 47,8 & 10 & 43,5 & 2 & 8,7 \\
\hline Confusão mental & 19 & 82,6 & 3 & 13,0 & 1 & 4,4 \\
\hline Pressão arterial sistólica $<90 \mathrm{mmHg}$ & 16 & 69,6 & 6 & 26,0 & 1 & 4,4 \\
\hline Saturação de oxigênio <93\% & 14 & 60,9 & 7 & 30,4 & 2 & 8,7 \\
\hline Volume urinário $<0,5 \mathrm{ml} / \mathrm{h} / \mathrm{kg}$ & 20 & 86,9 & 1 & 4,4 & 2 & 8,7 \\
\hline
\end{tabular}

*Um dos participantes não respondeu a essa questão; ${ }^{*}$ Dois dos participantes não responderam a essa questão. Fonte: Dados da pesquisa

Discussão

Nesta pesquisa, observou-se um predomínio de profissionais enfermeiros do sexo feminino, com idade média de 24,7 anos, graduados há no mínimo cinco anos e já titulados como especialistas, apresentando pelo menos um curso de pós-graduação. Destaca-se a ausência de vínculo prolongado entre os profissionais e os setores que atuavam no momento da pesquisa, sendo a maioria com uma atuação profissional nesse setor há menos de 12 meses. Tais achados assemelham-se às características da pesquisa realizada pelo Conselho Federal de Enfermagem (COFEN) que traçou o perfil da Enfermagem no Brasil, no qual mais da metade dos enfermeiros havia concluído a graduação há menos de 10 anos e possuíam algum curso de especialização [13].

No que tange ao conhecimento dos enfermeiros sobre os diferentes estágios clínicos da sepse, percebeu-se que grande parte dos profissionais (19-82,6\%) relatou ter conhecimento moderado sobre a temática. No entanto, ao deparar-se com a aplicabilidade de tais conhecimentos na identificação dos casos clínicos específicos, poucos foram os enfermeiros capazes de diferenciar corretamente os quadros de SIRS, sepse, sepse grave e choque séptico. Resultado semelhante foi encontrado em estudo que revelou um déficit de conhecimento baseado em evidência científica sobre o advento SIRS, Sepse, Sepse Grave e Choque Séptico entre enfermeiros intensivistas [11]. Na prática cotidiana a dificuldade em distinguir esses quadros patológicos pode estar diretamente relacionada com uma piora da qualidade da assistência e aumento da mortalidade dos pacientes [14].

Essa deficiência de conhecimento sobre a temática pode estar relacionada à formação acadêmica. Os enfermeiros participantes deste estudo, em sua maioria, (15- 65,2\%) afirmaram que receberam pouco conhecimento sobre sepse enquanto graduandos. Acredita-se que os conceitos de SIRS, sepse, sepse grave e choque séptico deveriam ser abordados de forma transversal nos cursos de graduação em enfermagem, uma vez que os futuros enfermeiros que atuarão em diferentes cenários clínicos poderão se deparar com pacientes com sepse e necessitarão de um aporte teórico para subsídio da prática. No entanto, pesquisa realizada com estudantes do último ano do curso de enfermagem demonstrou que os mesmos possuem pouco conhecimento sobre a temática, evidenciado pelo baixo número de acertos com relação às definições e aos sintomas de SIRS, sepse, sepse grave e choque séptico [12].

A equipe de enfermagem é a responsável pela manutenção do cuidado contínuo dos pacientes hospitalizados. Assim, o nível de conhecimento e a qualidade da assistência oferecida, sobretudo pelo enfermeiro, pode impactar diretamente o estado de saúde desses pacientes [15]. Nos casos de suspeita de sepse, vale ressaltar que o diagnóstico tardio pode levar a uma pior evolução do quadro clínico, culminando com o choque séptico [16]. Consequentemente, essa má evolução do quadro prolonga o tempo de internação e torna o paciente susceptível ao aumento de comorbidades como disfunções de órgãos, podendo aumentar o risco de mortalidade.

Tendo em vista a magnitude do problema da sepse e buscando uma melhor assistência aos portadores dessa síndrome, um grupo de especialistas de diferentes organizações se reúne periodicamente a cada quatro anos, desde 2004, para formular e 
atualizar a diretriz para o gerenciamento da sepse. Essa diretriz direciona o cuidado aos pacientes com sepse no âmbito hospitalar, a fim de subsidiar uma melhor prática [17]. Em 2016, foram propostas novas definições e critérios clínicos para a sepse e choque séptico e, terminologias como septicemia, síndrome séptica e sepse grave foram colocadas em desuso [18-19].

Pela nova diretriz, a sepse passa a ser compreendida como uma "disfunção orgânica potencialmente fatal causada por uma resposta imune desregulada a uma infecção". O choque séptico por sua vez, representa um quadro de "sepse acompanhada por profundas anormalidades circulatórias e celulares/metabólicas capazes de aumentar a mortalidade substancialmente" [18-20].

Para a identificação dos casos de sepse, os profissionais de saúde deverão se basear na suspeita ou presença confirmada de infecção associada ao aumento de dois pontos no Sequential Organ Failure Assessment (SOFA), que inclui dados clínicos e laboratoriais como: a razão entre a Pressão Parcial de Oxigênio Arterial $\left(\mathrm{PaO}_{2}\right)$ e a Fração Inalada de Oxigênio $\left(\mathrm{FiO}_{2}\right)$; Plaquetas; Bilirrubina; Pressão Arterial Média; Escala de Coma de Glasgow; Creatinina, Débito Urinário. Para o choque séptico, faz-se necessário além do quadro de sepse a utilização de drogas vasopressoras para a manutenção da pressão arterial média maior ou igual a 65 $\mathrm{mmHg}$ e lactato sérico maior que $2 \mathrm{mmol} / \mathrm{L}$ após a correta reanimação volêmica $[18,19]$.

Embora as novas definições tenham sido discutidas e apoiadas por muitos pesquisadores em todo o mundo, também geraram muita controvérsia. Dada a necessidade dos exames laboratoriais para avaliação do score SOFA, alguns pacientes podem ter o seu diagnóstico de sepse atrasado e, consequentemente, o seu tratamento. Um estudo realizado por pesquisadores vinculados ao ILAS e à Associação de Medicina Intensiva Brasileira (AMIB) teve como objetivo construir um consenso a respeito de como estas novas definições podem ser aplicadas no dia a dia. Para eles, a triagem de sepse em pacientes com suspeita de infecção, tanto no pronto-socorro quanto nas enfermarias, deve se basear em ferramentas sensíveis, destacando-se aquelas com base nos critérios de SIRS ou em qualquer disfunção orgânica clínica ou laboratorial. Devido aos diferentes contextos sociais acredita-se que o melhor equilíbrio entre a sensibilidade (SIRS) e a especificidade (disfunção orgânica) varie entre as diferentes instituições, dependendo da disponibilidade de recursos apropriados. Nesse sentido, o conhecimento dos enfermeiros sobre as definições adotadas em 2012 e até então utilizadas pode ser considerado fundamental para uma assistência de qualidade [21].

Apesar da magnitude da sepse e das recentes discussões sobre a identificação e manuseio dos casos, na presente pesquisa percebe-se que houve uma dificuldade por parte dos entrevistados na correta identificação dos casos apresentados. De forma semelhante, outro estudo, que buscou avaliar o conhecimento de profissionais de enfermagem e medicina acerca de sepse, sepse grave e choque séptico, identificou que o conhecimento dos profissionais sobre a temática é restrito [22].

Quanto ao reconhecimento de sinais e sintomas, destaca-se a questão da temperatura corporal, grande parte dos enfermeiros $(20-86,9 \%)$ reconhece a elevação da temperatura $\left(>38^{\circ} \mathrm{C}\right)$ como um sinal sugestivo de sepse e sepse grave, no entanto desconsideram a redução da temperatura $\left(<36^{\circ} \mathrm{C}\right)$ como uma variável importante a ser analisada. De forma semelhante, outro estudo identificou que $66,6 \%$ dos enfermeiros reconheceram a hipertermia como um sinal sugestivo da sepse. No entanto, apenas $11,1 \%$ reconhece a hipotermia como outro sinal sugestivo de sepse [11].

Outros sinais e sintomas que merecem atenção devido à baixa correlação por parte dos enfermeiros ao quadro de sepse referem-se à alteração da Frequência Cardíaca (> 90bpm) e a suspeita de infecção, as quais foram identificadas respectivamente por apenas $11(47,8 \%)$ e $10(43,5 \%)$ dos enfermeiros.

Destaca-se que a pouca familiaridade com a definição e com os sinais e sintomas de sepse, insere-se como uma característica que não está restrita aos enfermeiros. Um estudo brasileiro que avaliou o conhecimento dos médicos sobre sepse concluiu que eles apresentam dificuldade em reconhecer a sepse e sepse grave, provavelmente devido à dificuldade de reconhecer a disfunção de órgãos e a ausência de conhecimento de que a disfunção de órgãos define sepse grave [23].

$\mathrm{Na}$ identificação dos sinais e sintomas de sepse grave, enfermeiros tiveram dificuldade em correlacionar não apenas a baixa temperatura corporal, mas também a alteração dos níveis glicêmicos (> $110 \mathrm{mg} / \mathrm{dL}$ ) que esteve presente em apenas $11(47,8 \%)$ das respostas. Essa situação demonstra o desconhecimento e/ou pouca sensibilidade desses profissionais frente a dados objetivos obtidos rotineiramente nos serviços de saúde, de fácil mensuração e 
importância clínica, que compõem o rol dos problemas de enfermagem, e que são primordiais na detecção precoce de casos suspeitos de sepse.

Acredita-se que um dos motivos pelo qual a identificação da sepse ocorra tardiamente, refere-se ao déficit de conhecimento dos profissionais sobre as diretrizes clínicas e pelo fato de alguns dos seus sinais e sintomas não serem específicos, tais como: febre, taquicardia e taquipneia, os quais podem ser confundidos com outras patologias [7].

A não identificação correta por parte dos enfermeiros dos casos de pacientes com sepse em seus diferentes estágios pode implicar em uma pior evolução do quadro clínico dos pacientes e aumento dos gastos em saúde. Nesse sentido, alternativas para minimizar essa deficiência devem ser criadas, destacando-se a implementação de um programa de melhoria do desempenho para a sepse, conforme recomendado pelas Diretrizes Internacionais para o Gerenciamento de Sepse e Choque Séptico, publicado no ano de 2016[17]. Este programa deve envolver uma equipe multiprofissional e contar com a implantação de protocolos, coleta e avaliação de dados, além das ações de educação continuada direcionadas à aplicação das diretrizes na prática clínica $[24,25]$.

Acredita-se que a partir da reflexão sobre a realidade vivenciada pelos enfermeiros no atendimento aos pacientes sépticos e da atualização rotineira dos subsídios teóricos norteadores dessa assistência, possa haver uma ressignificação da prática diária com impacto significativo para a saúde dos pacientes. Esforços de gestores dos serviços de saúde e dos próprios profissionais aliados a instituições formadoras poderão auxiliar na disseminação de informações atualizadas sobre esse grande problema de saúde pública.

Este estudo tem como limitação o fato de restringir-se a avaliar os profissionais enfermeiros de uma única instituição hospitalar em um único momento. Acredita-se que seria importante extrapolar esta investigação para outras instituições e conduzir estudos com acompanhamento longitudinal a fim de identificar o comportamento desses profissionais no dia a dia da prática de enfermagem.

A maioria (19-82,6\%) dos enfermeiros deste estudo afirmou ter conhecimento moderado sobre a sepse e seus estágios clínicos. Percebeu-se que há dificuldade na aplicabilidade clínica dos conceitos inerentes à SIRS e aos diferentes estágios de sepse. Tal fragilidade pode estar associada à deficiência de formação na graduação, uma vez que a maioria dos enfermeiros $(15-65,2 \%)$ avaliou como pouco o conhecimento recebido sobre a temática enquanto graduandos.

A enfermagem é considerada a classe profissional que possui maior tempo na assistência direta com os pacientes, assumindo assim importância ímpar no reconhecimento precoce dos sinais de sepse. Nesse sentido, torna-se fundamental que os enfermeiros saibam reconhecer e distinguir precocemente os diferentes estágios de sepse, auxiliando a condução do tratamento e facilitando um desfecho favorável ao quadro clínico.

Vale destacar que a atualização dos profissionais configura-se como uma ferramenta imprescindível para a qualidade da assistência que deve ser cada vez mais encorajada nos diferentes serviços de saúde. Para tal faz-se necessária uma disposição das instituições formadoras, dos gestores e dos próprios profissionais na busca pelo conhecimento.

1. Instituto Latino-Americano para Estudos da Sepse. Sepse: um problema de saúde pública. Brasília; 2015. [citado 2017 Ago 10]. Disponível em: http://www.ilas.org.br/assets/arquivos/upload/Livro-ILAS(Sepse-CFM-ILAS).pdf

2. Torio CM, Andrews RM. National inpatient hospital costs: the most expensive conditions by payer, 2011: Statistical Brief \#160. 2013 Aug. In: Healthcare Cost and Utilization Project (HCUP) Statistical Briefs [Internet]. Rockville (MD): Agency for Healthcare Research and Quality (US); 2006 Feb. Disponível em: https://www.ncbi.nlm.nih.gov/books/NBK169005/

3. Vincent JL, Marshall JC, Namendys-Silva SA, François B, Martin-Loeches I, Pickkers P et al. Avaliação do fardo mundial das doenças críticas: a auditoria do Intensive Care Over Nations (ICON). Lancet Respir Med 2014;2(5):380-6.

4. Machado FR, Assunção MSC, Cavalcanti AB, Japiassú AM, Azevedo LCP, Oliveira MC. Chegando a um consenso: vantagens e desvantagens do Sepsis 3 considerando 
países de recursos limitados. Rev Bras Ter Intensiva 2016;28(4):361-5.

https://doi.org/10.5935/0103-507x.20160068

5. Giacomini MG, Lopes MVCAI, Gandolfi JV, Lobo SMA. Choque séptico: importante causa de morte hospitalar após alta da unidade de terapia intensiva. Rev Bras Ter Intensiva 2015;27(1):51-6. https://doi.org/10.5935/0103-507X.20150009

6. Dellinger RP, Levy MM, Rhodes A, Annane D, Gerlach H, Opa SM, et al. Campanha de sobrevivência à sepse: Diretrizes internacionais para tratamento de sepse grave e choque séptico: 2012. CCM Journal 2013;41(2):165-228.

7. Viana RAPP. Sepse, um problema de saúde pública: a atuação e colaboração da enfermagem na rápida identificação e tratamento da doença. 2 ed. São Paulo: CorenSP; 2017.

8. Bone RC, Balk RA, Cerra, FB, Dellinger RP, Fein AM, Knaus WA, et al. Definitions for sepsis and organ failure and guidelines for the use of innovative therapies in sepsis. The ACCP/SCCM Consensus Conference Committee. American College of Chest Physicians/ Society of Critical Care Medicine, 1992. Chest 1992;101(6):1644-55. https://doi.org/10.1378/chest.101.6.1644

9. Dutra CSK, Silveira LM, Santos AO, Pereira R, Stabile AM. Diagnósticos de enfermagem prevalentes no paciente internado com sepse no centro de terapia intensiva. Cogitare Enferm 2014;19(4):747-54. https://doi.org/10.5380/ce.v19i4.36801

10. Oliveira APC, Coelho MEAA, Almeida VCF, Lisboa KWSC, Macêdo ALS. Sistematização da assistência de enfermagem: implementação em uma unidade de terapia intensiva. Rev Rene 2012;13(3):601-12. https://doi.org/10.18471/rbe.v29i2.11707.s3374

11. Almeida APSR, Belchior PK, Lima MG, Souza LP. Conhecimento do profissional enfermeiro a respeito da sepse. Braz J Surg Clin Res 2013;4(4)5-10.

12. Santos JF, Alves AP, Stabile AM. Avaliação do conhecimento dos estudantes de enfermagem sobre sepse. Rev Eletr Enf 2012;14(4):850-6. https://doi.org/10.5216/ree.v14i4.15077

13. Machado MH, Wermelinger M, Vieira M, Oliveira E, Lemos W, Filho WA, et al. Aspectos gerais da formação da enfermagem: o perfil da formação dos enfermeiros, técnicos e auxiliares. Enferm Foco 2016;7(ESP):15-34. https://doi.org/10.21675/2357707x.2016.v7.nesp.687

14. Schmittz RK, Pelaes T, Paganini MC. Reconhecimento precoce do quadro de sepse em terapia intensiva: Atuação do enfermeiro. Revista Boletim de Enfermagem 2010;1:18-32.

15. Durthaler JM, Ernst FR, Johnston JA. Managing severe sepsis: a national survey of current practices. Am J Health Syst Pharm 2009;66(1):45-53. https://doi.org/10.2146/ajhp080067

16. Angus DC, Van der poll T. Severe sepsis and septic shock. Review. Erratum in: N Engl J Med 2013;369(21):2069-840-51.

17. Rhodes A, Evans LE, Alhazzani W, Levy MM, Antonelli M, Ferrer R, et al. Surviving sepsis campaign: international guidelines for management of sepsis and septic shock: 2016. Intensive Care Med 2017;45(3):486-552. https://doi.org/10.1007/s00134-0174683-6

18. Seymour CW, Liu VX, Iwashyna TJ, Brunkhorst FM, Rea TD, Scherag A, et al. Assessment of clinical criteria for sepsis: for the Third International Consensus Definitions for Sepsis and Septic Shock (Sepsis-3). JAMA 2016;315(8):801-10. https://doi.org/10.1001/jama.2016.0288

19. Shankar-hari M, Phillips GS, Levy ML, Seymour CW, Liu VX, Clifford S, et al. Developing a new definition and assessing new clinical criteria for septic shock for the Third International Consensus Definitions for sepsis and septic shock (Sepsis-3). JAMA 2016;315(8):775-87. https://doi.org/10.1001/jama.2016.0289

20. Singer M, Deutschman CS, Seymour CW, Shankar-Hari M, Annane D, Bauer M et al. The Third International Consensus Definitions for sepsis and septic shock (sepsis). JAMA 2016;315(8):801-10. https://doi.org/10.1001/jama.2016.0287

21. Machado FR, Assunção MSC, Cavalcanti AB, Japiassú AM, Azevedo LCP, Oliveira MC. Chegando a um consenso: vantagens e desvantagens do Sepsis3 considerando países de recursos limitados. Rev Bras Ter Intensiva 2016;28(4):361-5. http://dx.doi.org/10.5935/0103-507x.20170074 
22. Melech CS, Paganini MC. Avaliação do conhecimento de médicos e equipe de enfermagem nas ocorrências de sepse. Rev Med UFPR 2016;3(3):127-32. https://doi.org/10.5380/rmu.v3i3.47544

23. Assunção MSC, Corrêa TD, Bravim BA, Silva E. Como escolher os alvos terapêuticos para melhorar a perfusão tecidual no choque séptico. Einstein 2015;13(3):441-7. http://dx.doi.org/10.1590/S1679-45082015RW3148

24. Black MD, Schorr C, Levy MM. Knowledge translation and the multifaceted intervention in the intensive care unit. Crit Care Med 2012;40(4):1324-28. https://doi.org/10.1097/ccm.0b013e3182431673

25. Murphy DJ, Ogbu OC, Coopersmith CM. ICU director data: using data to assess value, inform local change, and relate to the external world. Chest 2015;147(4):1168-78. https://doi.org/10.1378/chest.14-1567 